\title{
Advances in Deep Learning in Mobile Interactive Algorithms and Learning Technologies
}

\author{
https://doi.org/10.3991/ijim.v14i10.15369 \\ Asadullah Shaikh $(\bowtie)$ \\ College of Computer Science and Information Systems, \\ Najran University, Najran, Saudi Arabia \\ asshaikh@nu.edu.sa
}

The Special Issue of the International Journal of Interactive Mobile Technologies (iJIM) is publishing very selective papers presented at the $6^{\text {th }}$ edition of International Conference on Communication, Management, and Information Technology- ICCMIT 2020. The ICCMIT 2020 was scheduled from 1-3 April 2020 in Athens, Greece which was postponed due to the COVID'19 outbreak, and the papers were presented online.

For this special issue, the ICCMIT 2020 received several papers from researchers, engineers, and industry professionals, from all around the world to publish their research in the field of science and technologies. But we selected best contributions from Russia, Romania, Cyprus, Saudi Arabia, Egypt, and Pakistan. These contributions are in the field of cellular automata, wireless sensor networks, mechatronic systems, deep learning, heterogeneous LTE-a networks, GPS services, web services, feature selection, data stream, mobile e-learning, traffic routing solution, and encoding systems.

The articles included in this Special Issue comprise different perspectives of deep learning in mobile interactive algorithms and learning technologies, presenting the diversity and actuality of this area.

The cellular networks especially the cellular automata approach and Global Positioning System (GPS) is widely used to control the traffic flow automatically using several methods. There are also new approaches to finding empty parking lots without GPS and providing the user with the exit for his parked car in indoor garages where thousands of cars are parked, and it is difficult to find the vehicle. In addition to the parking system, a novel solution is also proposed to estimate the routes and total time to travel in a transportation network. This solution optimizes Google's Map API using Open Street Map. These interesting facts and figures are published in the following articles.

Five articles present works developed on these topics:

- $\quad$ Modelling traffic on road junctions on parallel computing systems using cel-

lular automata approach

- $\quad$ Finding a Parked Car Location in a Multi-Story Building without GPS Service 
- A Four-Pronged Low Cost and Optimized Traffic Routing Solution

- Aerial mechatronic systems for collection of atmospheric and environmental data

- $\quad$ On-board video-evaluation algorithm of transverse safety clearance for ahead road-vehicle

With the intense use of the Internet of Things (IoT), systems are connected through Wireless Sensor Networks (WSN) and heterogeneous LTE networks. Accordingly, there is an ever-increasing number of network attacks. How does one identify WSN attacks and analyze the sensor data? The new mobile communication technologies propose many solutions for assuring the prevention from attacks for advanced real-time applications

Three contributions illustrate works in these areas:

- Anomaly Detection in Wireless Sensor Networks: A Proposed Framework

- Modified Proportional Fair Scheduling Algorithm for heterogeneous LTE-A Networks

- Optimization of Velocity Mode In Buslaev Two - Contours Networks Via Competition Resolution Rules

The availability of E-Learning and mobile learning through powerful tools based on deep learning was always present. But after the COVID'19 outbreak, the need for Elearning has increased rapidly. The next stage of E-Learning systems is usability. The usability of Moodle and Blackboard systems among university students has been addressed. After usability, the next concern is to identify the learning style of the learner and provide the recommended learning strategy that fits each learner individually.

Three articles present works in these areas:

- A Study on the Usability of Moodle and Blackboard-Saudi Students Perspectives

- Implementing Web Services using PHP Soap Approach

- Energy Consumption Prediction Using Deep Learning Technique: Case Study of Computer College

Social networking using mobile and web services become an essential element of daily life and these networks are widely used as communication platforms in the world of business. Social networking software such as Twitter, Facebook, and WhatsApp offer valuable opportunities for collaboration due to their open nature. Therefore, there is an intense need for a novel framework using feature selection techniques to discover the characteristics of these social networks. For example, WhatsApp does not have Chabot's, whereas their competitors from Telegram have them already built in. These interesting facts and figures are published in the following articles. 
Two submissions illustrate works in these areas:

- E Characterize: A novel feature selection-based framework for characterizing entrepreneurial influencers in Arabic Twitter

- Messaging System Design Based on Using Servers and Encoding System

The DataStream is the huge amount of information created in different fields, in social media activities and Internet of Things applications, Such information cannot be handled through traditional data mining algorithms because of constraints like limited memory, data speed, and dynamic environment

An article that describes the contribution of data stream models for concept drift detection provides a major contribution to the field of classification of models. These methods work by monitoring the classifier's predictions:

- CDDM: Concept Drift Detection Model for Data Stream

This article illustrates the concept drift detection model that monitors the accuracy of the classification model.

\section{Author}

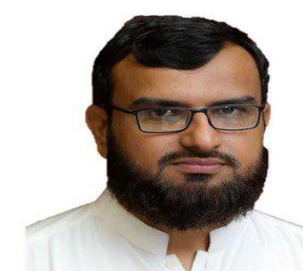

Asadullah Shaikh received a Ph.D. degree in software engineering from the University of Southern Denmark, an M.Sc. degree in software engineering and management from Goteborg University, Sweden, and the B.Sc. degree in software development from the University of Huddersfield, England. He is currently working as Associate Professor, Head of research, and Coordinator of seminars and training with the College of Computer Science and Information Systems, Najran University, Najran, Saudi Arabia. He has more than 80 publications in the area of software engineering in international journals and conferences. He has vast experience in teaching and research. His current research topics are UML model verification, UML class diagrams verification with OCL constraints for complex models, formal verification, feedback technique for unsatisfiable UML/OCL class diagrams. He has worked as a Researcher in UOC Barcelona Spain. He is also the Editor of the International Journal of Advanced Computer Systems and Software Engineering (IJACSSE) and on the International Advisory Board of several conferences and journals. Dr. Shaikh is also a guest editor of several special issues that are indexed in ISI Web of Science and Scopus. Further details can be obtained using www.asadshaikh.com 